Therefore $X$ parametrizes a connected open subset of the hyperbola $H: x^{2} / a^{2}-$ $y^{2} / b^{2}=1$.

For a unit-speed parametrization $X$ of a hyperbola $F: x^{2} / a^{2}-y^{2} / b^{2}=1$, it is easy to show that $X$ satisfies condition (C). This completes the proof of the theorem.

ACKNOWLEDGMENT. The authors would like to express their deep thanks to the referee who kindly provided suggestions and to Professor Jeongseon Baek for valuable advice that improved this note.

\title{
REFERENCES
}

1. M. P. do Carmo, Differential Geometry of Curves and Surfaces, Prentice-Hall, Englewood Cliffs, NJ, 1976.

2. R. S. Millman and G. D. Parker, Elements of Differential Geometry, Prentice-Hall, Englewood Cliffs, NJ, 1977.

Department of Mathematics, Chonnam National University, Kwangju 500-757, Korea dosokim@chonnam.ac.kr

Department of Mathematics, Kyungpook National University, Taegu 702-701, Korea yhkim@knu.ac.kr

\section{A Class of Dirichlet Series Integrals}

\section{Jonathan M. Borwein}

1. PRELIMINARIES. In this note we extend the solution to a recent MonTHLY problem to analyze a broad class of Dirichlet series and illustrate the result in action in various ways. More precisely, in [6] the following integral evaluation is obtained:

$$
\int_{0}^{\infty} \frac{\{3-2 \sqrt{2} \cos (t \log 2)\}|\zeta(1 / 2+i t)|^{2}}{t^{2}+1 / 4} d t=\pi \log 2
$$

This somewhat recondite-looking result transpires to be a case of a rather pretty class of evaluations given in Theorem 1 , and worth making explicit.

We let $\lambda(s):=\sum_{n>0} \lambda_{n} n^{-s}$ represent a formal Dirichlet series, with real coefficients $\lambda_{n}$ and we set $s:=\sigma+i \tau$ with $\sigma=\Re(s)>0$. We refer to [2], [3], and [7] for other, largely standard, notation. We consider the following integral

$$
\iota_{\lambda}(\sigma):=\int_{0}^{\infty}\left|\frac{\lambda(s)}{s}\right|^{2} d \tau,
$$

as a function of the Dirichlet series $\lambda$ (i.e., of the coefficients $\lambda_{n}$ ).

The question of evaluating (2) when $\lambda$ is the alternating zeta-function was posed and solved (at least formally) in [6]. It is convenient to know that for strictly positive $u$ and $a$

$$
\int_{0}^{\infty} \frac{\cos (a t)}{t^{2}+u^{2}} d t=\frac{\pi}{2 u} e^{-a u}
$$

as may be established by contour integration, by consulting a computer algebra system, or otherwise. 


\section{THE MAIN RESULT}

Theorem 1. For $\lambda(s)=\sum_{n=1}^{\infty} \lambda_{n} n^{-s}$ and $s=\sigma+i \tau$ with fixed $\sigma=\Re(s)>0$ such that the Dirichlet series is absolutely convergent it is true that

$$
\iota_{\lambda}(\sigma)=\int_{0}^{\infty}\left|\frac{\lambda(s)}{s}\right|^{2} d \tau=\frac{\pi}{2 \sigma} \sum_{n=1}^{\infty} \frac{\Lambda_{n}^{2}-\Lambda_{n-1}^{2}}{n^{2 \sigma}}
$$

where $\Lambda_{n}:=\sum_{k=1}^{n} \lambda_{k}$ and $\Lambda_{0}:=0$.

More generally, for given absolutely convergent Dirichlet series $\alpha(s):=\sum_{n=1}^{\infty} \alpha_{n} n^{-s}$ and $\beta(s):=\sum_{n=1}^{\infty} \beta_{n} n^{-s}$

$$
\Re \int_{0}^{\infty} \frac{\alpha(s) \bar{\beta}(s)}{\sigma^{2}+\tau^{2}} d \tau=\frac{\pi}{2 \sigma} \sum_{n=1}^{\infty} \frac{A_{n} B_{n}-A_{n-1} B_{n-1}}{n^{2 \sigma}},
$$

in which $A_{n}=\sum_{k=1}^{n} \alpha_{k}$ and $B_{n}=\sum_{k=1}^{n} \beta_{k}$.

Proof. Let $\lambda_{N}(s)=\sum_{n=1}^{N} \lambda_{n} n^{-s}$. Then

$$
\begin{aligned}
\int_{0}^{\infty} \frac{\left|\lambda_{N}(s)\right|^{2}}{\tau^{2}+\sigma^{2}} d \tau=\int_{0}^{\infty} \frac{\sum_{N \geq n, m>0} \lambda_{n} \lambda_{m} n^{-\sigma+i \tau} m^{-\sigma-i \tau}}{\tau^{2}+\sigma^{2}} d \tau \\
\quad=2 \sum_{N \geq n>m>0} \frac{\lambda_{n} \lambda_{m}}{(n m)^{\sigma}} \int_{0}^{\infty} \frac{(n / m)^{i \tau}}{\tau^{2}+\sigma^{2}} d \tau+\sum_{n=m=1}^{N} \frac{\lambda_{n} \lambda_{m}}{(n m)^{\sigma}} \int_{0}^{\infty} \frac{(n / m)^{i \tau}}{\tau^{2}+\sigma^{2}} d \tau \\
\quad=2 \sum_{N \geq n>m>0} \frac{\lambda_{n} \lambda_{m}}{(n m)^{\sigma}} \int_{0}^{\infty} \frac{\cos (\tau \log (n / m))}{\tau^{2}+\sigma^{2}} d \tau+\sum_{n=1}^{N} \frac{\lambda_{n}^{2}}{n^{2 \sigma}} \int_{0}^{\infty} \frac{1}{\tau^{2}+\sigma^{2}} d \tau \\
\quad=\frac{\pi}{\sigma} \sum_{N \geq n>m>0} \frac{\lambda_{n} \lambda_{m}}{(n m)^{\sigma}(n / m)^{\sigma}}+\frac{\pi}{2 \sigma} \sum_{n=1}^{N} \frac{\lambda_{n}^{2}}{n^{2 \sigma}} \\
\quad=\frac{\pi}{\sigma} \sum_{n=1}^{N} \frac{\lambda_{n} \Lambda_{n}}{n^{2 \sigma}}-\frac{\pi}{2 \sigma} \sum_{n=1}^{N} \frac{\lambda_{n}^{2}}{n^{2 \sigma}}=\frac{\pi}{2 \sigma} \sum_{n=1}^{N} \frac{\Lambda_{n}^{2}-\Lambda_{n-1}^{2}}{n^{2 \sigma}} .
\end{aligned}
$$

Next, we observe that $\left|\lambda_{N}(s)\right|^{2} \leq\left(\sum_{n=1}^{\infty}\left|\lambda_{n}\right| n^{-\sigma}\right)^{2}=M<\infty$, where $M$ is independent of $\tau$. Hence

$$
\left|\frac{\lambda_{N}(s)}{s}\right|^{2} \leq \frac{M}{\tau^{2}+\sigma^{2}},
$$

and (4) follows from Lebesgue's theorem on dominated convergence when $N \rightarrow \infty$. We can establish (5) in a similar fashion.

By Dirichlet's test, the final series in (4) is convergent when $\sigma>0$ provided that $\left\{\Lambda_{n}\right\}$ is bounded, but we cannot automatically guarantee that it is equal to the integral in (4) in this case, or even that the integral is finite. Simple continuation arguments just will not work.

It is easy to check that

$$
\langle\alpha, \beta\rangle_{\tau}:=\int_{0}^{\infty} \frac{\alpha(s) \bar{\beta}(s)}{\sigma^{2}+\tau^{2}} d \tau
$$


defines an extended-value inner product on the space of Dirichlet series with $\langle\alpha, \alpha\rangle_{\sigma}=$ $\iota_{\alpha}(\sigma)$, which is typically finite for $\sigma$ large enough.

Next, let $\mathrm{L}_{\mu}(s):=\sum_{n=1}^{\infty}\left(\frac{\mu}{n}\right) n^{-s}$ denote the primitive $L$-function corresponding to the Legendre-Jacobi-Kronecker symbol $\left(\frac{\mu}{n}\right)$ [5]. An imprimitive character sum modulo $m$ is induced by another character sum modulo $m^{\prime}$ for which $m \mid m^{\prime}$ and $\chi(m)=\chi^{\prime}(n$ $\bmod m^{\prime}$ ). We remind the reader that, for a prime $p$ and $\mu$ relatively prime to $p$, the Legendre symbol $\left(\frac{\mu}{p}\right)$ is 1 when $\mu$ is a quadratic residue modulo $p$ and is -1 otherwise. The Jacobi symbol extends this multiplicatively for $\mu$ the product of distinct primes, while the Kronecker symbol provides the final extension to all relatively prime pairs $\mu$ and $n$.

Example 1. First, we note that with $\lambda \equiv 1$ we recover the arctangent formula

$$
\int_{R} \frac{1}{\sigma^{2}+t^{2}} d t=\frac{\pi}{\sigma}
$$

Similarly, let $\vartheta:=s \mapsto\left(1+2^{-s}\right)^{-1}$. Here again $\frac{\sigma}{\pi} \iota_{\vartheta}(\sigma)=\frac{1}{2} \vartheta(2 \sigma)$.

Example 2. For the Riemann zeta-function $\left(\zeta=\mathrm{L}_{1}\right)$ and for $\sigma>1$,

$$
\frac{\sigma}{\pi} \iota_{\zeta}(\sigma)=\zeta(2 \sigma-1)-\frac{1}{2} \zeta(2 \sigma),
$$

as $\lambda_{n}=1$ and $\Lambda_{n}=n$. By contrast it is known-see [4, eqn. (69)] or [1]-that on the critical line

$$
\frac{1 / 2}{\pi} \iota_{\zeta}\left(\frac{1}{2}\right)=\log (\sqrt{2 \pi})-\frac{1}{2} \gamma .
$$

More broadly, Crandall (in unpublished notes) has shown that when $0<\sigma<1$

$$
\iota_{\zeta}(\sigma)=\pi \int_{0}^{1} \sum_{n \geq 0} \frac{\theta^{2}}{(n+\theta)^{1+2 \sigma}} d \theta=\pi \int_{0}^{1} \theta^{2} \zeta(1+2 \sigma, \theta) d \theta,
$$

where $\zeta(s, a)$ is the Hurwitz zeta-function, which is easy to compute. Working a little harder [1], we obtain

$$
\frac{\sigma}{\pi} \iota_{\zeta}(\sigma)= \begin{cases}-\frac{1}{2}[\zeta(2 \sigma)+2 \zeta(2 \sigma-1) /(2 \sigma-1)] & \text { if } 0<\sigma<1 \\ -\frac{1}{2}[\zeta(2 \sigma)-2 \zeta(2 \sigma-1)], & \text { if } 1<\sigma<\infty\end{cases}
$$

Example 3. There are similar formulas for $s \mapsto \zeta(s-k)$ with $k$ integral. For instance, applying the result in (4) with $\zeta_{1}$ the function sending $t \mapsto \zeta(t+1)$ yields

$$
\frac{1}{\pi} \int_{0}^{\infty} \frac{|\zeta(3 / 2+i \tau)|^{2}}{1 / 4+\tau^{2}} d \tau=\frac{1}{\pi} \iota_{\zeta_{1}}\left(\frac{1}{2}\right)=2 \zeta(2,1)+\zeta(3)=3 \zeta(3),
$$

on using Euler's result $[3]$ that $\zeta(2,1):=\sum_{n} 1 / n^{2} \sum_{k=1}^{n-1} 1 / k=\zeta(3)$.

Example 4. For the classical alternating zeta-function, $\alpha(s):=\left(1-2^{1-s}\right) \zeta(s)$, we recover (see [6]): 


$$
\frac{\sigma}{\pi} \iota_{\alpha}(\sigma)=\frac{1}{2} \alpha(2 \sigma)
$$

as $\lambda_{n}=(-1)^{n+1}=\Lambda_{n}^{2}-\Lambda_{n-1}^{2}$. Set $\sigma=1 / 2$. Then

$$
\begin{aligned}
\frac{|\alpha(1 / 2+i t)|^{2}}{1 / 4+t^{2}} & =\frac{\left|1-2^{1 / 2-i t}\right|^{2}|\zeta(1 / 2+i t)|^{2}}{1 / 4+t^{2}} \\
& =(3-2 \sqrt{2} \cos (t \ln (2))) \frac{|\zeta(1 / 2+i t)|^{2}}{1 / 4+t^{2}}
\end{aligned}
$$

is precisely the integrand in (1). Thus, since $\alpha(2 \sigma)=\log 2$, we see that $\iota_{\alpha}(1 / 2)=$ $\pi \log 2$ is the evaluation in [6]. Note that to justify the exchange of sum and integral implicit in (4) we have to group the terms more carefully as $1 / 2$ is below the abscissa of absolute convergence of the series-or use the approach in [1] on the Hurwitz zetafunction.

Example 5. For the Catalan zeta-function, $\beta:=\mathrm{L}_{-4}$ :

$$
\frac{\sigma}{\pi} \iota_{\beta}(\sigma)=\frac{1}{2} \beta(2 \sigma)
$$

as $\lambda_{2 n}=0, \lambda_{2 n+1}=(-1)^{n}$, and again $\Lambda_{n}^{2}-\Lambda_{n-1}^{2}=\lambda_{n}$.

For $\mathrm{L}_{-3}$, the same pattern holds, in that $\frac{\sigma}{\pi} \iota_{\mathrm{L}_{-3}}(\sigma)=\frac{1}{2} \mathrm{~L}_{-3}(2 \sigma)$, but it fails for $\mathrm{L}_{5}, \mathrm{~L}_{-7}$, and so on. In general $\mathrm{L}_{ \pm d}$ does not lead to output that is again a primitive L-series modulo $d$. For example,

$$
\frac{\sigma}{\pi} \iota_{L_{5}}(\sigma)=-\sum_{5 \nmid n} \frac{(-1)^{(n \bmod 5)}}{n^{2 \sigma}} .
$$

and

$$
\frac{\sigma}{\pi} \iota_{\mathrm{L}_{-8}}(\sigma)=\mathrm{L}_{-8}(2 \sigma)-\frac{1}{2} \mathrm{~L}_{-4}(2 \sigma) .
$$

Unlike the case of primitive L-series (meaning that the coefficients are not induced by restriction of another $L$-series), these right-hand side series have coefficients that are not multiplicative, though always the coefficients repeat modulo $d$.

While (4) gives an effective way of evaluating the integral, direct numerical computation to high precision presents a more interesting challenge. This is in part because of the severe oscillations of the integrand- shown in Figure 1 with $\sigma=1 / 2,1$, and 2 for $\alpha$ and $L_{-3}$, respectively. In large part, the issue lies in estimating the integrand well, and so is intrinsically nontrivial.

3. A CHARACTERIZATION AND SOME REWORKINGS. The preceding results are nicely explained by the following theorem:

Theorem 2. The unique Dirichlet series with all nonvanishing coefficients satisfying the functional equation

$$
\frac{\pi}{\sigma} \iota_{\vartheta}(\sigma)=\frac{1}{2} \vartheta(2 \sigma)
$$



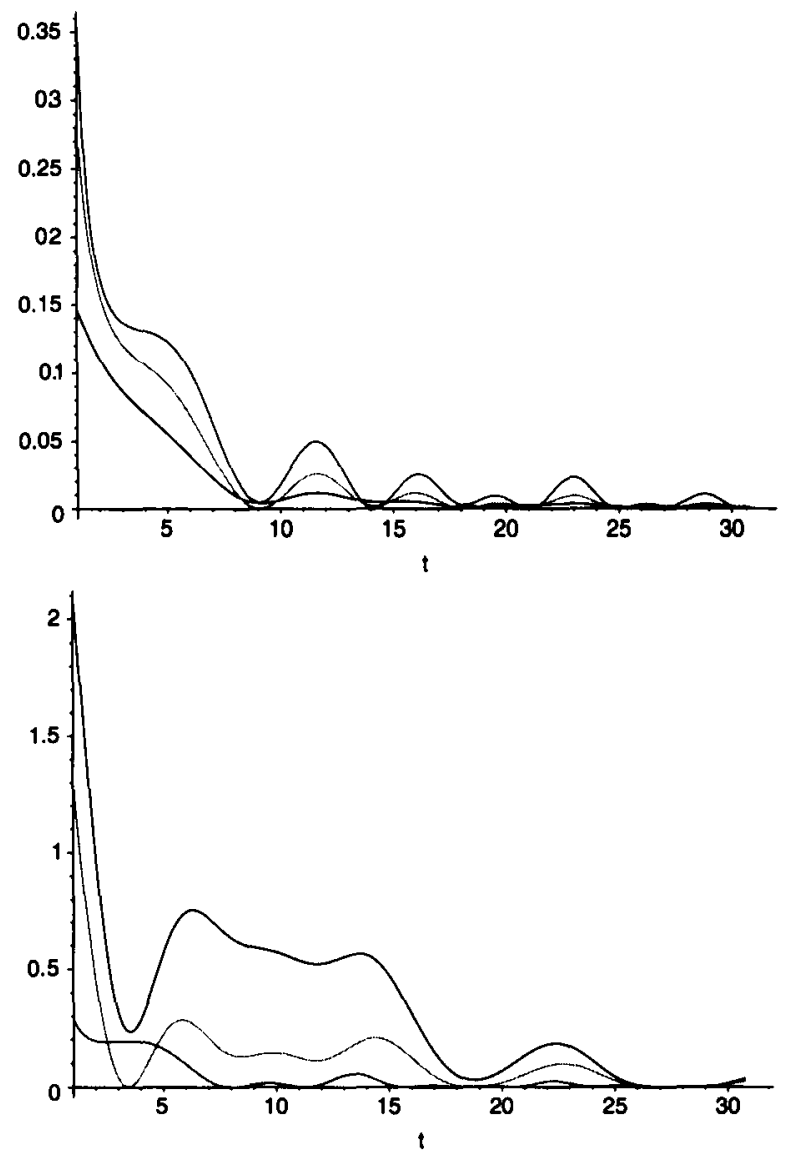

Figure 1. The integrands for $\alpha$ and $L_{-3}$ respectively with $\sigma=1 / 2,1$ and 2

is the alternating zeta-function $\alpha$. Moreover, each solution is alternating of the form

$$
\vartheta(s)=\sum_{k=1}^{\infty} \frac{(-1)^{k}}{n_{k}^{s}}
$$

for an increasing sequence of integers $0<n_{1}<n_{2}<\cdots<n_{k} \cdots$.

Proof. Comparing coefficients and invoking (4), we see that, in the ring of formal Dirichlet series $\frac{\sigma}{\pi} \iota \psi(\sigma)=\frac{1}{2} \psi(2 \sigma)$ if and only if

$$
\Psi_{n}^{2}-\Psi_{n-1}^{2}=\psi_{n}\left(\Psi_{n-1}+\Psi_{n}\right)=\frac{1}{2} \psi_{n}
$$

for all $n$, where as before $\Psi_{n}:=\sum_{m=1}^{n} \psi_{m}$. Equivalently, we have either $\Psi_{n}=\Psi_{n-1}$ or $\Psi_{n}+\Psi_{n-1}=1$ for all $n$. Observing that $\Psi_{0}=0$, we deduce that $\Psi_{n}=0$ or 1 for each $n$.

This leads to (7) when reexpressed in terms of $\psi_{n}$.

Note that $\lambda \equiv 1$ and $s \mapsto 1-3^{-s}$ have the requisite form, as do $\mathrm{L}_{-3}, \mathrm{~L}_{-4}$, and $\vartheta$ in the previous examples, whereas the other functions therein do not. 
The derivation of (4) can be adjusted to produce

$$
\int_{0}^{\infty}\left|\frac{\lambda(s)}{s}\right|^{2} d \tau+\frac{\pi}{2 \sigma} \sum_{n>0} \frac{\lambda_{n}^{2}}{n^{2 \sigma}}=\iota_{\lambda}(\sigma)+\frac{\pi}{2 \sigma} \sum_{n>0} \frac{\lambda_{n}^{2}}{n^{2 \sigma}}=\frac{\pi}{\sigma} \sum_{n>0} \frac{\lambda_{n} \Theta_{n}}{n^{2 \sigma-1}} .
$$

Here $\Theta_{n}:=\frac{1}{n} \sum_{m=1}^{n} \lambda_{m}$ is the average order of $\lambda_{n}$ [7].

For an interesting variety of arithmetic functions, both $\lambda(\sigma)$ and

$$
\lambda_{2}(\sigma):=\sum_{n>0} \lambda_{n}^{2} n^{-\sigma}
$$

have closed forms (see [7, chaps. 16-18], [5]) and the discussion to follow, so (8) can be viewed as giving information about the more recondite average order series on the far right. Thus, an application of the arithmetic-geometric mean inequality-for $\lambda_{n} / n^{\sigma}$ and $n \Theta_{n} / n^{\sigma}$-yields

$$
\int_{0}^{\infty}\left|\frac{\lambda(s)}{s}\right|^{2} d \tau=\frac{\pi}{\sigma} \sum_{n>0} \frac{\lambda_{n} \Theta_{n}}{n^{2 \sigma-1}}-\frac{\pi}{2 \sigma} \sum_{n>0} \frac{\lambda_{n}^{2}}{n^{2 \sigma}} \leq \frac{\pi}{2 \sigma} \sum_{n>0} \frac{\Theta_{n}^{2}}{n^{2 \sigma-2}} .
$$

Thus,

$$
\frac{\pi}{\sigma} \sum_{n>0} \frac{\lambda_{n} \Theta_{n-1}}{n^{2 \sigma-1}} \leq \int_{0}^{\infty}\left|\frac{\lambda(s)}{s}\right|^{2} d \tau \leq \frac{\pi}{2 \sigma} \sum_{n>0} \frac{\Theta_{n}^{2}}{n^{2 \sigma-2}} .
$$

Moreover, we have

$$
\left.\left|\frac{\pi}{\sigma} \sum_{n>0} \frac{\lambda_{n} \Theta_{n}}{n^{2 \sigma-1}}-\int_{0}^{\infty}\right| \frac{\lambda(s)}{s}\right|^{2} d \tau \mid \leq \frac{\pi}{2 \sigma} \sum_{n>0} \frac{\lambda_{n}^{2}}{n^{2 \theta}}=\frac{\pi}{2 \sigma} \lambda_{2}(2 \sigma),
$$

where both quantities on the left are nonnegative.

Example 6. Clearly, $\left(\mathrm{L}_{ \pm d}\right)_{2}$ is very simple. In particular, for $p$ prime we have $\left(\mathrm{L}_{ \pm p}\right)_{2}(s)=\left(1-p^{-s}\right) \zeta(s)$.

Let $\sigma_{k}$ denote the $k$ th power sum of divisors of $n$. A beautiful formula [7, Theorem 291] asserts that:

$$
\sum_{n=1}^{\infty} \frac{\sigma_{k}(n)}{n^{s}}=\zeta(s) \zeta(s-k) \quad(\Re(s)>\max \{1, k+1\})
$$

while [7, Theorem 305] gives the following remarkable identity:

$$
\sum_{n=1}^{\infty} \frac{\sigma_{a}(n) \sigma_{b}(n)}{n^{s}}=\frac{\zeta(s) \zeta(s-a) \zeta(s-b) \zeta(s-a-b)}{\zeta(2 s-a-b)}
$$

when $\Re(s)>1+\max \{a, b, a+b\}$. This result, which is due to Ramanujan for $a=b$, yields $\left(\mathrm{L}_{\sigma_{a}}\right)_{2}$.

Example 7. By contrast, for the Möbius function [7], [8] we have

$$
\mu(s)=\sum_{n>0} \mu(n) n^{-s}=1 / \zeta(s)
$$


-directly from the Möbius inversion formula. Here $\mu(n)=(-1)^{k}$ if $n$ has $k$ distinct prime factors and is zero otherwise. On the other hand, $\mu_{2}(s)=\sum_{n \in \mathrm{SF}} n^{-s}$, where SF represents the squarefree numbers, has no explicit formula. From (11) with $M(n):=$ $\sum_{k=1}^{n} \mu(k)$, we obtain

$$
\left.\left|\frac{\pi}{\sigma} \sum_{n>0} \frac{\mu_{n} M_{n}}{n^{2 \sigma}}-\int_{0}^{\infty}\right| \frac{1}{s \zeta(s)}\right|^{2} d \tau \mid \leq \sum_{n \in \mathrm{SF}} \frac{1}{n^{2 \sigma}} .
$$

Recall that the Mertens conjecture-that always $|M(n)| \leq \sqrt{n}$ holds for all $n$-was only recently shown to fail for some $n$ no smaller than $10^{12}$ and probably larger that $10^{30}[8$, pp. 207-210].

DEDICATION. This article is dedicated

To the memory of "Sir Edward Wright, the former principal and vice-chancellor of Aberdeen University who died on February 2 [2005] aged 98, [and who] became one of the country's most distinguished mathematicians, despite being largely self-taught."1

ACKNOWLEDGEMENTS. The research of the author is supported by NSERC and by the Canada Research Chair Programme.

\section{REFERENCES}

1. D. Borwein and J. M. Borwein, Van der Pol expansions of L-series, Canad. Math. Bull. (to appear).

2. J. M. Borwein and D. H. Bailey, Mathematics by Experiment. Plausible Reasoning in the 21st Century, AK Peters, Natick, MA, 2003.

3. J. Borwein, D. Bailey, and R. Girgensohn, Experimentation in Mathematics. Computational Paths to Discovery, AK Peters, Natick, MA, 2004.

4. J. M. Borwein, D. M. Bradley and R. E. Crandall, Computational strategies for the Riemann zeta-function, J. Comp. and Appl. Math, 121 (2000), 247-296; also available as CECM Preprint 98:118.

5. J. M. Borwein and K.-K. (Stephen) Choi, On Dirichlet series for sums of squares, Ramanujan J., 7 (2003) 195-238.

6. A. Ivic, Solution to Monthly Problem \#10939, proposed by A. Ivic, with his solution in this MONTHLY 110 (2003), 847-848.

7. G. H. Hardy and E. M. Wright, 4th ed, An Introduction to the Theory of Numbers, Oxford University Press, Oxford, 1959.

8. J. Havel, Gamma: Exploring Euler's Constant, Princeton University Press, Princeton, 2003.

Faculty of Computer Science, Dalhousie University, Halifax, Nova Scotia B3H IW5, Canada jborwein@cs.dal.ca

\footnotetext{
${ }^{1}$ ww. telegraph.co.uk/news/main. Jhtml? xml-/news/2005/02/10/db1001.xmlksSheet= /portal/2005/02/10/1xportal.html
} 